\title{
Comportamientos de riesgo para la salud en niños y adolescentes con intentos de suicidio y en sus familiares
}

\author{
MÓNICA EDITH BELLA
}

\section{Risk factors and behaviors among children and adolescents hospitalized for a suicidal attempt}

Background: Suicidal behavior is the result of the interaction of several factors and represents an increasing health problem. Aim: To characterize epidemiologically children and adolescents with suicidal attempts and compare them to a control group. Material and Methods: A case-control and prospective study, based on clinical interviews and tests performed to 30 children and adolescents hospitalized due to a suicidal attempt and their parents or tutors, (GIS) and 40 ambulatory controls without such history (CG). Psychopathological disorders, risk behaviors (substance use, maltreatment, peer violence, sexual abuse and early sexual relations), family factors and their association with suicidal attempts, were analyzed. Results: Seventy seven percent of participants with a history of suicidal attempt had psychopathological disorders (depression in 38\%) and a higher prevalence of suicidal behavior, maltreatment, substance and sexual abuse. Male sex was a risk factor for suicide among children under 12 years and female sex was a risk factor for adolescents aged over 12 years. Other risk factors detected were a risky communication between parents and siblings. Conclusions: Children and adolescents who attempted suicide and their families have a higher prevalence of risk behaviors than the control group.

(Rev Med Chile 2012; 140: 1417-1424).

Key words: Dangerous behavior; Suicidal ideation; Suicide, attempted; Psychopathology; Violence.

Hospital de Niños de la
Santísima Trinidad de
Córdoba. Profesora de
la Facultad de Medicina
Universidad Nacional de
Córdoba y Universidad
Católica de Córdoba.
Argentina.
Conflicto de interés: nada
que declarar
Recibido el 23 de enero
de 2012, aceptado el 9 de
julio de 2012.
Correspondencia a
Dra. Mónica Edith Bella
Hospital de Niños de la
Santísima Trinidad de
Córdoba
Avenida Ferroviarios
esquina Av. Pucará.
Tel/fax:0351-4586427.
E-mail: bellamonica@
hotmail.com.

Hospital de Niños de la Santisima Trinidad de la Facultad de Medicin Universidad Nacional de Córdoba y Universida Católica de Córdoba. Argentina.

Conflicto de interés: nada declarar

Recibido el 23 de enero de 2012, aceptado el 9 de Correspondencia a Dra. Mónica Edith Bella Hospital de Niños de Córdoba Avenida Ferroviarios hotmail.com.
L a infancia y adolescencia es una etapa de la vida de baja morbi-mortalidad, pero entre las causas más importantes que afectan la salud de este grupo etario se encuentran los comportamientos suicidas, los accidentes y los comportamientos de riesgo para la salud como son el uso de sustancias adictivas, la violencia y los comportamientos de riesgo sexual ${ }^{1-4}$. Los estudios refieren que estos problemas de salud muestran una tendencia en aumento y esto es motivo de preocupación para la mayor parte de los países ya que afecta el desarrollo y la calidad de vida de las personas más jóvenes ${ }^{1,4,5}$.

La morbi-mortalidad ocasionada por la violencia, suicidio, homicidios y accidentes constituyen un grave problema de salud pública para los países de la región de las Américas ${ }^{4,6-8}$. En Argentina la tasa de suicidio en el grupo etario de 10 a 25 años aumentó de 1,5 cada 100.000 habitantes en 1990 a 8,2 cada 100.000 habitantes en el $2008^{6,9}$.

La bibliografía acuerda respecto a los comportamientos suicidas en que son actos complejos que responden a la interacción de factores psicopatológicos, familiares y sociales. Entre los factores psicopatológicos, la depresión y los trastornos de conducta, son los más prevalentes ${ }^{10-14}$. Con respecto a las variables familiares y sociales los estudios asocian los comportamientos suicidas en los familiares como el factor de mayor riesgo; otras características también reconocidas son la 
familia monoparental y las relaciones conflictivas padres-hijos ${ }^{15-18}$.

Se estima que por cada adolescente que muere por suicidio hay entre 15 y 20 intentos no mortales realizados y que la mayor parte de ellos no desean realmente la muerte. Las muertes ocasionadas por los suicidios constituyen un motivo de preocupación para la salud infantojuvenil, ya que este tipo de muertes son evitables y prevenibles cuando se conocen sus causas y se interviene oportunamente. La mayor parte de las medidas de prevención han estado orientadas a los aspectos individuales (detección y tratamiento de los trastornos psicopatológicos) y en el grupo etario de los mayores de 16 años, siendo pocos los estudios que abordan la problemática de los menores de esta edad ${ }^{19-25}$.

De lo expuesto deriva la importancia de efectuar estudios en esta población a fin de determinar los factores intervinientes en esta conducta. El objetivo de la presente investigación fue:

- Caracterizar epidemiológicamente a los niños/ adolescentes con intento de suicidio y compararlos con un grupo control, analizando trastornos psicopatológicos, comportamientos de riesgo para la salud (uso de sustancias, maltrato, violencia con pares, abuso sexual y relaciones sexuales), características familiares y su asociación con los intentos de suicidio infantojuvenil.

- Identificar y establecer la relevancia que tienen las variables estudiadas en los intentos de suicido de los niños/adolescentes.

\section{Material y Método}

El estudio fue de casos y control, observacional, analítico y prospectivo. Se efectuó en el Hospital de Niños de la Santísima Trinidad que es el centro de salud de mayor complejidad en pediatría en el sistema provincial. El período de estudio fue de un año, entre los meses de enero y diciembre, y se efectuó en base a entrevista clínica semiestructurada y al test, "Cómo es tu-su familia" realizado a los niños/adolescentes y a sus padres/tutores. El test es un instrumento creado por la Organizacion Panamericana de la Salud que se encuentra validado en el país y en la población en estudio ${ }^{19,26,27}$.

Para cumplimentar con el objetivo propuesto en la investigación se conformaron dos grupos de estudio:
- El grupo de casos (GIS): se conformó con todos los niños/adolescentes que se hospitalizaron por intento de suicidio durante el período en que se desarrolló la investigación (30 casos). Fueron excluidos de este grupo 4 niños/adolescentes que fueron hospitalizados en el mismo período pero que sus padres no aceptaron participar de la evaluacion familiar, test "Cómo es tu-su familia", quedando conformado finalmente el grupo con 26 casos.

- El grupo control (GC) se constituyó con 40 niños/adolescentes que fueron selecionados de modo aleatorio en los consultorios externos de los Servicios de Medicina del Deporte y Odontología en el período de ingreso de los casos. El criterio de incorporación de los controles era tener la misma edad y sexo de los casos, ser población consultante en la misma institución y estar acompañado por uno de sus padres quien participaba de la entrevista familiar.

Para establecer el tamaño de la muestra, por cada caso que ingresó, se seleccionaron controles en una relación de 1 a 1,5, estableciéndose un nivel de seguridad de $95 \%$ y un poder estadístico de $80 \%$.

La participación de los niños/adolescentes y de sus padres/tutores en la investigación fue voluntaria. Se les explicó el objetivo de la investigación y la metodología a utilizar, certificándose la conformidad mediante la firma del consentimiento informado.

Para valorar las variables propuestas para el estudio se efectuaron entrevistas clínicas y se tomó el test "Cómo es tu-su familia" a los niños/ adolescentes y a sus padres o tutores. El test es un instrumento que tiene una estructura de opciones múltiples que permite caracterizar sociodemograficamente a la población, determinar conductas de riesgo para la salud en los niños/ adolescentes y en sus familiares, obtener estructura, funcionamiento familiar y situaciones de estrés ${ }^{19}$. El test fue administrado por un evaluador.

Las variables analizadas fueron definidas operativamente como:

- Intento de suicidio: todo acto realizado por la persona con la intención de quitarse la vida, sin llegar a tener como resultado la muerte.

- Comportamientos de riesgo para la salud: acciones que implican una mayor probabilidad que se produzcan consecuencias negativas para la salud. Se valoró: intento de suicidio previo, 
uso de sustancias en el último mes (tabaco, alcohol, marihuana, otras drogas), sentimiento de tristeza permanente, recibir golpes o maltrato, violencia con pares, abuso sexual y relaciones sexuales.

- Caraterización familiar. Para cumplimentar este objetivo se tomó el test "Cómo es tu-su familia" que permite evaluar estructura, funcionamiento familiar y situaciones de estrés familiar por medio de preguntas con opciones múltiples.

- Las situaciones de estrés familiar: eventos que ocurrieron en la familia en el último año y que el niño/adolescente identifica que lo han afectado emocionalmente en forma relevante identificando: muerte de familiar, problemas de dinero, problemas familiares, separación de los padres, problemas escolares, violencia en el hogar, cambio de vivienda y fuga del hogar.

Los datos se procesaron estadísticamente mediante análisis bivariado y multivariado. Para los datos categóricos se utilizó análisis de frecuencias y ANOVA para las variables numéricas. Los procedimientos utilizados fueron test de $\square^{2}$, Mantel Haenzel o test de Fisher obteniéndose los Odds Ratio (OR) y los Intervalos de Confianza (IC). En todos los casos se estableció un nivel de significación de $\mathrm{p}<0,05$.

El protocolo de estudio de esta investigación fue aprobado por el Comité de Investigaciones del Hospital de Niños de la Santísima Trinidad y por el Comité Institucional de Ética de Investigación en Salud del Polo Hospitalario del Ministerio de Salud de la Provincia de Córdoba.

\section{Resultados}

El 69\% de los niños/adolescentes hospitalizados por intento de suicidio (GIS) fueron mujeres y el $31 \%$ varones y sus edades estaban comprendidas entre los 8 y los 16 años con una media de edad de 13,38 años $( \pm 0,40)$. La media de edad mostró diferencias significativas entre los sexos expresando en varones una media de 12,13 años $(\mathrm{p}<0,03)$ $\mathrm{y}$ en mujeres de 13,94 años. El grupo control se conformó con $55 \%$ mujeres y $45 \%$ varones y sus edades estaban entre 8 y 16 años con una media de edad de 12,36 $( \pm 0,52)$ las mujeres y de 12,71 $( \pm 0,53)$ los varones. El 84,62\% $(\mathrm{n}=22)$ de los casos del GIS estaba escolarizado y $15,38 \%(n=4)$ abandonó el colegio en el último año (75\% asistía al secundario y $25 \%$ al primario). En el GC todos los casos se encontraban escolarizados. Con respecto a la presencia de actividades extraescolares, $11,5 \%(\mathrm{n}=3)$ del GIS realizaba (danzas y deportes) y $97,5 \%(\mathrm{n}=39)$ del GC.

El 76,92\% del GIS presentaban trastornos psicopatológicos detectando: trastornos del humor (depresión mayor y episodios depresivos de trastorno bipolar) $38,46 \%(\mathrm{n}=10)(\mathrm{p}<0,0001)$, trastorno en el desarrollo de la personalidad 19,23\% $(\mathrm{n}=5)$, trastornos de conducta $15,38 \%(\mathrm{n}=4)$ y trastorno psicótico 3,85\% $(\mathrm{n}=1)$. El 23,08\% $(\mathrm{n}=6)$ de los casos no presentaban un trastorno psicopatológico y presentaban problemas relacionados con la crianza del niño/adolescente. En el GC no se detectaron trastornos psicopatológicos.

Al estudiar la presencia de tratamientos psicológicos o psiquiátricos previos a la hospitalización se encontró que $73,08 \%$ de los casos no había recibido tratamiento y $26,92 \%$ se encontraba en tratamiento en el momento del intento de suicidio.

Con el test se valoraron los comportamientos de riesgo para la salud en los niños/adolescentes, detectando como significativos en el GIS: uso de tabaco 53,85\% ( $<<0,0001$, OR $=22,17)$, alcohol 26,92\% ( $<<0,005)$, sentimientos de tristeza permanente $76,92 \%(\mathrm{p}<0,001, \mathrm{OR}=5,56)$, violencia con pares $50 \%(\mathrm{p}<0,008, \mathrm{OR}=7,00)$, maltrato $34,62 \%(\mathrm{p}<0,005, \mathrm{OR}=6,53)$ y otros. Los resultados del GIS y GC se expresan en Tabla 1. Al comparar de la variable tristeza con uso de alcohol se detectó que se asoció al 100\% de los casos $(\mathrm{p}<0,05)$.

Con respecto a los comportamientos de riesgo para la salud explorados en los familiares del GIS se encontraron: intentos de suicidio y suicidios en $53,85 \%(p<0,002, O R=5,50)$, violencia con pares $38,45 \%(\mathrm{p}<0,01, \mathrm{OR}=4,38)$ y abuso sexual $19,23 \%(\mathrm{p}<0,06, \mathrm{OR}=4,52)$. Los resultados del GIS y GC se expresan en la Tabla 2.

En relación a la estructura familiar el GIS mostró: familia biparental 34,62\% $(\mathrm{n}=9)$, familia monoparental 30,77\% $(\mathrm{n}=8)(\mathrm{p}<0,005)$ materna $87,5 \%$ y paterna $12,5 \%$, madre y pareja $19,23 \%(\mathrm{n}=$ 5) y otras estructuras familiares $15,39 \%(\mathrm{n}=4)$. En el GC el 77,50\% $(\mathrm{n}=31)(\mathrm{p}<0,005)$ familia biparental, el 12,50\% $(n=5)$ familia monoparental materna, madre y pareja $7,50 \%(n=3)$ y padre y pareja $2,50 \%(n=1)$. Con respecto al funcionamiento familiar se encontró como significativo 
Tabla 1. Comportamientos de riesgo para la salud en niños y adolescentes con intentos de suicidio (GIS) y en grupo control (GC)

\begin{tabular}{|c|c|c|c|c|c|}
\hline $\begin{array}{l}\text { Comportamientos de } \\
\text { riesgo para la salud }\end{array}$ & $\begin{array}{c}\text { GIS } \\
\begin{array}{c}\text { Frecuencia en } \% \\
n=26\end{array}\end{array}$ & $\begin{array}{c}\text { GC } \\
\begin{array}{c}\text { Frecuencia en } \% \\
\mathbf{n}=\mathbf{4 0}\end{array}\end{array}$ & *Valor p & $\begin{array}{l}\text { Odds } \\
\text { Ratio }\end{array}$ & IC95\% \\
\hline Intento de suicidio o suicidio & $19,23 \%(n=5)^{*}$ & $0 \%$ & $p<0,001$ & - & - \\
\hline Uso de tabaco & $53,85 \%(n=14)^{*}$ & $5 \% \quad(n=2)$ & $p<0,0001$ & 22,17 & $5,01-97,98$ \\
\hline Uso de alcohol & $26,92 \%(n=7)$ * & $0 \%$ & $p<0,005$ & - & - \\
\hline Uso de sustancias (marihuana) & $7,69 \%(n=2)^{*}$ & $0 \%$ & $p<0,05$ & - & - \\
\hline Uso de otras drogas & $3,85 \%(n=1)$ & $0 \%$ & - & - & - \\
\hline Sentimientos de tristeza & $76,92 \%(n=20)^{*}$ & $37,50 \%(n=15)$ & $p<0,001$ & 5,56 & $1,88-16,44$ \\
\hline Violencia con pares & $50 \% \quad(n=13)^{*}$ & $12,50 \%(n=5)$ & $p<0,008$ & 7,00 & $2,17-22,62$ \\
\hline Relaciones sexuales & $50 \% \quad(n=13)^{*}$ & $2,50 \%(n=1)$ & $p<0,0001$ & 38,80 & $8,17-84,35$ \\
\hline Abuso sexual & $15,38 \%(n=4)^{*}$ & $0 \%$ & $p<0,01$ & - & - \\
\hline Recibir golpes o maltrato & $34,62 \%(n=9)^{*}$ & $7,50 \%(n=3)$ & $p<0,005$ & 6,53 & $1,69-25,20$ \\
\hline
\end{tabular}

Tabla 2. Comportamientos de riesgo para la salud en familiares del grupo con intento de suicidio (GIS) y del grupo control (GC)

\begin{tabular}{|c|c|c|c|c|c|}
\hline $\begin{array}{l}\text { Comportamientos de riesgo } \\
\text { de salud en familiares }\end{array}$ & $\begin{array}{l}\text { Familiares GIS } \\
\text { Frecuencia en } \% \\
\qquad \mathbf{n}=\mathbf{2 6}\end{array}$ & $\begin{array}{c}\text { Familiares GC } \\
\text { Frecuencia en } \% \\
\text { n }=40\end{array}$ & *Valor p & $\begin{array}{l}\text { Odds } \\
\text { Ratio }\end{array}$ & IC $95 \%$ \\
\hline Intento de suicidio o suicidio & $53,85 \%(n=14)^{*}$ & $17,50 \%(n=7)$ & $p<0,002$ & 5,50 & $1,84-16,44$ \\
\hline Uso de tabaco & $73,08 \%(n=19)$ & $67,50 \%(n=27)$ & - & - & - \\
\hline Uso de alcohol & $38,46 \%(n=10)$ & $(n=16)$ & - & & - \\
\hline Uso de sustancias (marihuana) & $15,38 \%(n=4)$ & $(n=8)$ & - & - & - \\
\hline Uso de otras drogas & $15,38 \%(n=4)^{*}$ & $(n=4)$ & $p<0,5$ & 1,64 & $0,40-6,69$ \\
\hline Uso de tranquilizantes & $11,54 \%(n=3)$ & $(n=6)$ & - & - & - \\
\hline Uso de solventes & $15,38 \%(n=4)$ & $(n=4)$ & - & - & - \\
\hline Sentimientos de tristeza & $50 \% \quad(n=13)^{*}$ & $(n=16)$ & $p<0,5$ & 1,50 & $0,56-3,99$ \\
\hline Violencia con pares & $38,46 \%(n=10)^{*}$ & $12,50 \%(n=5)$ & $p<0,01$ & 4,38 & $1,34-16,68$ \\
\hline Abuso sexual & $19,23 \%(n=5)^{*}$ & $5 \% \quad(n=2)$ & $p<0,06$ & 4,52 & $0,93-22,08$ \\
\hline Recibir golpes o maltrato & $30,77 \%(n=8)^{*}$ & $22,50 \%(n=9)$ & $p<0,4$ & 1,53 & $0,52-4,55$ \\
\hline
\end{tabular}

en el GIS una comunicación padre-hijo y madrehijo de alto riesgo. Los resultados del GIS y GC se expresan en la Tabla 3.

$\mathrm{Al}$ analizar las situaciones estresantes referidas por los niños/adolescentes se expresaron como significativas en el GIS los problemas escolares $61,54 \%(\mathrm{p}<0,001)$, violencia en la familia $26,93 \%$ $(\mathrm{p}<0,005)$ y fuga del hogar $19,23 \%(\mathrm{p}<0,006)$. Los resultados de los dos grupos se exponen en la Tabla 4.
Se efectuaron diferentes análisis multivariado de correspondencias múltiples a fin de detectar las variables que se asociaba con mayor riesgo al grupo con intento de suicidio y el modelo que mostró mejor ajuste expresó en el eje uno el uso de sustancias, el abuso sexual, el uso de alcohol, el maltrato y los intentos de suicidios previos en los niños/adolescentes y en el eje dos el abuso sexual, el uso de sustancias, el maltrato y los intentos de suicidio y suicidios en los familiares (Figura 1). 
Tabla 3. Caracterización de las relaciones familiares en el grupo con intento de suicidio (GIS) y en el grupo control (GC)

\begin{tabular}{|c|c|c|c|c|c|c|}
\hline & \multicolumn{3}{|c|}{$\begin{array}{l}\text { Comunicación padre-hijo/a } \\
\text { Frecuencia en } \% \text { y n }=26\end{array}$} & \multicolumn{3}{|c|}{$\begin{array}{l}\text { Comunicación madre-hijo/a } \\
\text { Frecuencia en } \% \text { y } n=40\end{array}$} \\
\hline & No riesgo & Riesgo & Alto riesgo & No riesgo & Riesgo & Alto riesgo \\
\hline GIS & $\begin{array}{l}42,31 \% \\
(n=11)\end{array}$ & $\begin{array}{l}15,38 \% \\
(n=4)\end{array}$ & $\begin{array}{l}42,31 \% * \\
(n=11)\end{array}$ & $\begin{array}{c}65,38 \\
(n=17)\end{array}$ & $\begin{array}{l}15,38 \% \\
(n=4)\end{array}$ & $\begin{array}{l}19,23 \% * \\
(n=5)\end{array}$ \\
\hline GC & $\begin{array}{c}80 \% \\
(n=32)\end{array}$ & $\begin{array}{c}10 \% \\
(n=4)\end{array}$ & $\begin{array}{c}10 \% \\
(n=4)\end{array}$ & $\begin{array}{l}82,50 \% \\
(n=33)\end{array}$ & $\begin{array}{c}10 \% \\
(n=4)\end{array}$ & $\begin{array}{l}7,50 \% \\
(n=3)\end{array}$ \\
\hline
\end{tabular}

*Valor de $p<0,004 \square^{2}$ de Pearson y $\square^{2}$ MV-G2.

Tabla 4. Situaciones de estrés en niños y adolescentes con intentos de suicidio y en grupo control

\begin{tabular}{|c|c|c|c|c|c|}
\hline \multirow[b]{2}{*}{ Muerte de familiar } & \multicolumn{2}{|c|}{$\begin{array}{c}\text { GIS } \\
\begin{array}{c}\text { Frecuencia en } \% \\
n=26\end{array}\end{array}$} & \multicolumn{2}{|c|}{$\begin{array}{c}\text { GC } \\
\text { Frecuencia en } \% \\
n=40\end{array}$} & \multirow{2}{*}{$\begin{array}{c}\text { * Valor } \mathbf{p} \\
-\end{array}$} \\
\hline & $50 \%$ & $(n=13)$ & $30 \%$ & $(n=12)$ & \\
\hline Problemas escolares & $61,54 \%$ & $(n=16)^{*}$ & $22,50 \%$ & $(n=9)$ & $p<0,001$ \\
\hline Problemas familiares & $42,31 \%$ & $(n=11)^{*}$ & $12,50 \%$ & $(n=5)$ & $p<0,02$ \\
\hline Separación de padres & $7,69 \%$ & $(n=2)$ & $5 \%$ & $(n=2)$ & - \\
\hline Violencia en la familia & $26,93 \%$ & $(n=7)^{*}$ & $7,50 \%$ & $(n=3)$ & $p<0,005$ \\
\hline Problemas económicos & $73,08 \%$ & $(n=19)^{*}$ & $32,50 \%$ & $(n=13)$ & $p<0,04$ \\
\hline Cambio de vivienda & $26,93 \%$ & $(n=7)^{*}$ & $7,50 \%$ & $(n=3)$ & $p<0,005$ \\
\hline Fuga del hogar & $19,23 \%$ & $(n=5)^{*}$ & $0 \%$ & & $p<0,006$ \\
\hline
\end{tabular}

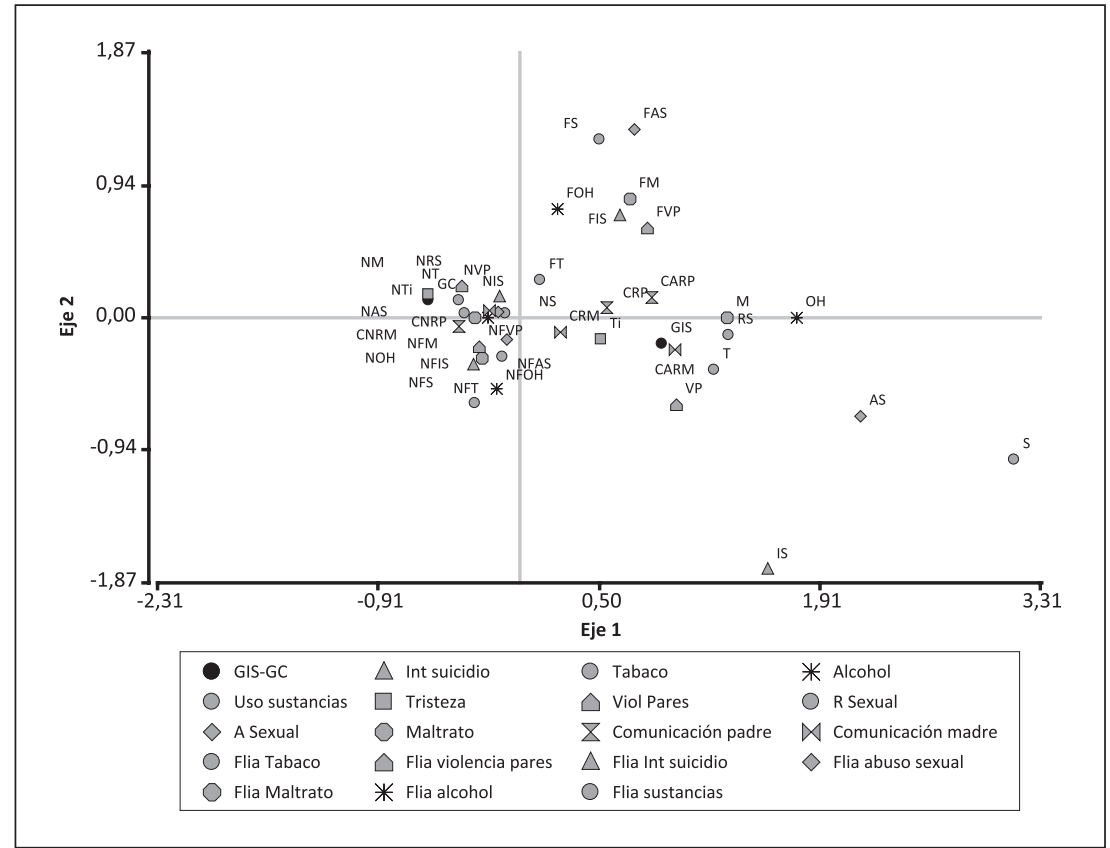

Figura 1. Análisis multivariado de los comportamientos de riesgo para la salud y comunicación con su padre y su madre en niños/adolescentes y familiares de los dos grupos estudiados (GIS y GC). 


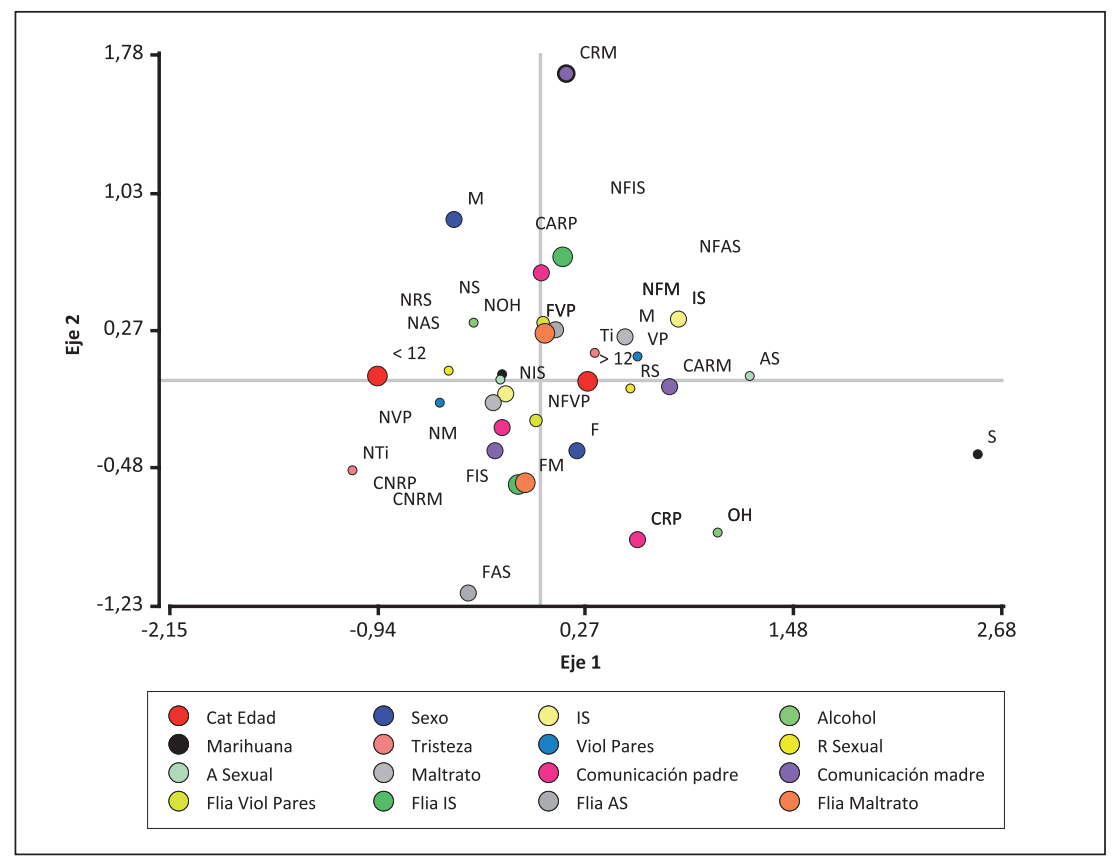

Figura 2. Análisis multivariado de los factores de riesgo asociados a los intentos de suicidio en los niños/adolescentes mayores y menores de 12 años.
Al categorizar al GIS en dos grupos etarios, mayores y menores de 12 años, el análisis multivariado mostró que a los menores de 12 años se asoció el sexo masculino ( $\mathrm{p}<0,02)$, y a los mayores de 12 años el sexo femenino, el uso de sustancias (alcohol-marihuana), comunicación de riesgo con padre-madre y el abuso sexual en los adolescentes y en sus familiares (Figura 2).

\section{Discusión}

Los comportamientos suicidas de los niños/ adolescentes del grupo etario estudiado son complejos y responden a la interrelación de factores biológicos, psicológicos y sociales como los detectados en este estudio, en el que se encontró además de trastornos psicopatológicos y comportamientos suicidas previos una mayor prevalencia de comportamientos violentos (maltrato, violencia con pares, abuso sexual) y uso de sustancias, tanto en los niños/adolescentes como en los familiares del GIS.

Respecto a los comportamientos violentos se observó que se asociaron 6 veces más al GIS y es de señalar que los valores de maltrato y abuso sexual detectados para este grupo superan a los del GC que mostró una prevalencia similar a la referida en los estudios efectuados en la población esco- larizada ${ }^{1,28,29}$. Entre las consecuencias que genera el vivir en hogares con problemas de violencia se reconocen los sentimientos de tristeza, angustia, ansiedad, pérdida de esperanza, alteraciones en el desarrollo de la personalidad y sexualidad y uso de sustancias entre otros ${ }^{28-32}$, y estas características también se encontraron asociadas a los niños/ adolescentes del GIS. Además en este aspecto, los niños/adolescentes identificaron a las situaciones de violencia en el hogar como un factor de estrés importante para ellos.

Con respecto a las variables relacionadas con la sexualidad, se observó que el abuso sexual fue más prevalente en los niños/adolescentes y familiares del GIS y se asoció con mayor riesgo al grupo mayor de 12 años. La presencia de estos factores de riesgo conlleva con frecuencia a alteraciones en el desarrollo psicosexual, relaciones sexuales tempranas, embarazos no deseados y enfermedades de trasmisión sexual también expresados algunos de ellos en este grupo ${ }^{28-30}$.

Otro de los comportamientos de riesgo explorados, el uso de sustancias, mostró que tabaco, alcohol y marihuana fueron las más utilizadas. Aun cuando los valores de consumo detectados para estas sustancias son similares a los referidos para la población escolarizada en este grupo etario, en el GIS se observó un comportamiento por 
sexo diferente al descripto en las investigaciones afectando más a las mujeres ${ }^{33-36}$. A diferencia del GIS, el GC mostró un comportamiento por sexo acorde al descripto en los estudios mostrando una baja prevalencia en el uso de sustancias y esto probablemente se relaciona con la elevada frecuencia de actividad deportiva de los niños/adolescentes de este grupo, expresándose esto como un factor de protección ${ }^{33-36}$.

En relación a los aspectos familiares estudiados, los comportamientos violentos (suicidio, maltrato y abuso) y la comunicación de riesgo con los padres se expresaron como variables asociadas a los intentos de suicidio infantojuvenil, y esta situación se asoció con mayor riesgo al grupo mayor de 12 años.

Los comportamientos suicidas son un motivo de preocupación para orientar el cuidado de la salud de los más jóvenes y las medidas de prevención en su mayor parte han estado dirigidas al diagnóstico y tratamiento oportuno de los trastornos psicopatológicos infantojuveniles. Pero la presente investigación muestra que para el grupo menor de 16 años se deberían contemplar también acciones preventivas familiares y comunitarias (escuela, grupos de pares) que tengan en cuenta los comportamientos de riesgo detectados. En este aspecto, la violencia, en sus diferentes modos de presentación (maltrato, violencia con pares y abuso sexual), es un factor de riesgo que se asoció fuertemente a los comportamientos suicidas de los niños/ adolescentes. A partir de lo expuesto se puede expresar:

- Los niños/adolescentes con intentos de suicidio presentan mayor prevalencia de trastornos psicopatológicos, intentos de suicidio previos, uso de sustancia, violencia con pares, maltrato, abuso sexual, relaciones sexuales tempranas, familias monoparentales y relaciones de riesgo con los padres.

- Los familiares de los niños/adolescentes con intento de suicidio tienen mayor frecuencia de comportamientos de riesgo para la salud y esto incrementa el riesgo de comportamientos suicidas en este grupo etario.

- En los menores de 12 años el sexo masculino se expresó como un factor de riesgo que se asoció al intento de suicidio y en los mayores de 12 años el sexo femenino, el uso de sustancias, el abuso sexual, el maltrato y la comunicación de riesgo con los padres.

\section{Referencias}

1. Concha-Eastman A, Krug E. Informe mundial sobre la salud y la violencia. Rev Panam Salud Pública 2002; 12 (4): 227-9.

2. Blum R. The health of young people in a global context. J Adolesc Health 2004; 35: 402-18.

3. Madaleno M, Morello P, Espindola Infante F. Salud y desarrollo de adolescentes y jóvenes en Latinoamérica y El Caribe: desafíos para la próxima década. Salud Pública de México 2003; 45 (1): 132-9.

4. Organización Mundial de la Salud. El suicidio un problema de salud pública enorme y sin embargo prevenible. Disponible en: http://www.who.int/mediacentre/ news/releases/2004/pr61/es/index.html [Consultado el 20/04/2011]

5. Duarte CS, Bordin I, Paula CS, Hoven CW. Conductas suicidas en adolescentes brasileños: análisis exploratorio de prevalencia y factores de riesgo. Rev Psiquiatría y Salud Integral 2002; 2 (4): 32-7.

6. Serfaty E, Foglia L, Masaútis A, Negri G. Mortalidad por causas violentas en adolescentes y jóvenes 10-24 años. Argentina 1991-2000. Vertex 2007; 40-8.

7. Llorca Castro F, Ortún Rubio V. Mortalidad innecesariamente prematura y sanitariamente evitable en Costa Rica. Rev Esp Salud Pública 2010; 84: 771-87.

8. Borges G, Orozco R, Benjet C, Medina-Mora M. Suicidio y conductas suicidas en México: retrospectiva y situación actual Salud Pública Mex 2010; 52 (4): 292-304.

9. Bella ME, López de Neira M, Villace MB, Acosta L, Luchesse M, Gallo V, et al. Tendencia de muertes por suicidio en adolescentes y jóvenes de Argentina 2000-2008. Rev. XI Jornadas de Investigación Científica. Facultad de Medicina UNC 2010; 11-6.

10. Spirito A, Valeri S, Boergers J, Donaldson D. Predictors of continued suicidal behavior in adolescents following a suicide attempt. J Clin Child Adolesc Psychol 2003; 32 (2): 284-9.

11. Olfson M, Gameroff M, Marcus S, Greenberg T, Shaffer D. National trends in hospitalization of youth with intentional self-inflected injuries. Am J Psychiatric 2005; 162: 1328-35.

12. D'Eramo KS, Prinstein MJ, Freeman J, Grapentine WL, Spirito A. Psychiatric diagnoses and comorbidity in relation to suicidal behavior among psychiatrically hospitalized adolescents. Child Psychiatry Hum Dev 2004; 35: 21-35.

13. Pacheco B, Lizana C, Celhay I. Diferencias clínicas entre adolescentes hospitalizados por intento suicida y adolescentes hospitalizados por otra causa psiquiátrica. Rev Med Chile 2010; 138: 160-7. 
14. Vairo C, Ruiz M, Blanco F, et al. Adolescentes y comportamiento suicida. Factores de riesgo. Rev Arg Psiquiatría Vértex 1999; 14: 179-89.

15. Gould M, Greenberg T, Velting DM, Shaffer D. Youth suicide risk and preventive interventions: a review of the past 10 years. J Am Acad Child Adolesc Psychiatry 2003; 42 (4): 386-405.

16. Rutter, PA, Behrendt AE. Adolescent suicide risk: four psychosocial factors. Adolescence 2004; 39: 295-302.

17. Roy A. Combination of family history of suicidal behavior and childhood trauma may represent correlate of increased suicide risk. Journal of Affective Disorders. 2011; 130: 205-8.

18. Pavez P, Santander N, Carranza J, Vera-Villarroel. Factores de riesgo familiares asociados a la conducta suicida en adolescentes con trastorno depresivo. Rev Med Chile 2009; 137: 226-33.

19. Bella ME, Fernández AR, Acevedo G, Willington JM. Socio-demographic and psychopathological analysis of child and adolescent attempted suicides. Vertex 2008; 19 (78): 10-6.

20. Sourander A, et al. Childhood predictors of completed and severe suicide attempts: findings from the Finnish 1981 Birth Cohort Study. Arch Gen Psychiatry 2009; 66 (4): 398-406.

21. Eisenberg ME, Ackard DM, Resnick MD. Protective factors and suicide risk in adolescents with a history of sexualabuse. J Pediatr 2007; 151 (5): 482-7.

22. Salzinger S, Rosario M, Feldman RS, Ng-Mak DS. Adolescent suicidal behavior: associations with preadolescent physical abuse and selected risk and protective factors. J Am Acad Child Adolesc Psychiatry 2007; 46 (7): 859-66.

23. Jakobsen IS, Christiansen E, Larsen KJ, Waaktaar T. Differences between youth with a single suicide attempt and repeaters regarding their and their parents history of psychiatric illness. Arch Suicide Res 2011; 15 (3): 265-76.

24. Parellada $\mathrm{M}$, et al. Is attempted suicide different in adolescent and adults? Psychiatry Res 2008; 15 (1-3): 131-57.

25. Groholt BMD, Oivind E. Suicide among children and younger and older adolescent in Norway: A comparative study. J. Am. Acad. Child Adolescent. Psychiatry 1998; 37 (5): 473-81.

26. Organización Panamericana de la Salud. Washington D.C. Familia y Adolescencia. Indicadores de Salud. Manual de aplicación de instrumentos e instrumento abreviado. 1999.

27. Santis R, Hidalgo CG, Hayden V, Anselmo E, Rodríguez
J, Cartajena J, et al. Consumo de sustancias y conductas de riesgo en consumidores de pasta base de cocaína y clorhidrato de cocaína no consultantes a servicios de rehabilitación. Rev Med Chile 2007; 135: 45-53.

28. Espinoza Gómez F, Pamplona V, Hernández Sánchez OA, Plasencia-García G. Violencia doméstica y riesgo de conducta suicida en universitarios adolescentes. Salud Pública de México 2010; 52 (3): 213-9.

29. Senn TE, Carey MP, Vanable PA. Childhood and adolescent sexual abuse and subsequent sexual risk behavior: evidence from controlled studies, methodological critique, and suggestions for research. Clin Psychol Rev 2008; 28 (5): 711-35.

30. Houston J, Murphy J, Adamson G, Stringer M, Shevln M. Childhood sexual abuse, early cannabis use, and psychosis: Testing an interaction model based on the national comorbidity survey. Schizophrenia Bulletin 2008; 34 (3): 580-8.

31. Marks DJ, Miller SR, Schulz KP, Newcorn JH, Halperin JM. The interaction of psychosocial adversity and biological risk in childhood aggression. Psychiatry Res 2007; 151: 221-30.

32. Brunstein Klomec A, Marroco F, Kleiman M, Schonfed IS, Gould MS. Bullying, depression, and suicidality in adolescents. J Am Acad Child Adolesc Psychiatry 2007; 46 (1): 40-9.

33. Ganz D, Sher L. Suicidal behavior in adolescents with comorbid depression and alcohol abuse. Minerva Pediatr 2009; 61 (3): 333-47.

34. Ocampo R, Bojorquez L, Cortés M. Consumo de sustancias y suicidios en México: resultados del Sistema de Vigilancia Epidemiológica de las Adicciones, 1994-2006. Rev Salud Pública de México 2009; 51 (4): 306-12.

35. Observatorio Argentino de Drogas (OAD): Resumen ejecutivo. Disponible en: http://www.asociacionantidroga.org.ar/superint/Estadisticas/Observatorio Argentino de Drogas-resumen actualizado febrero 07. [Consultado 15/07/2011].

36. Paulone I, Candioti CA. Consumo de sustancias adictivas en adolescentes escolarizados. Arch Argent Pediatr 2006; 104 (3): 227-33.

37. Haavet OR, Straand J, Saugstad OD, Grunfeld B. Illness and exposure to negative life experiences in adolescence: two sides of the same coin? A study of 15-year-olds in Oslo, Norway. Acta Pediatric 2004; 93 (3): 405-11.

38. Saffer D, Pfeffer C. Practice Parameter for the Assessment and Treatment of Children and Adolescents with Suicidal. Behavior. American Academy of Child and Adolescent Psychiatry. J Am Acad Child Adolesc Psychiatry 200140 (7): 24-51. 